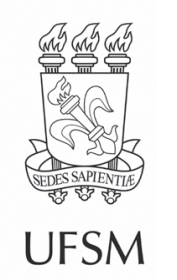

Artigos

\title{
Diferentes modelos para o afilamento do tronco de Eucalyptus sp. para o cenário florestal brasileiro
}

\section{Different models for Eucalyptus sp. trunk taper for the brazilian forestry scenario}

\author{
Lucas Kröhling Bernardi ${ }^{\oplus}$ \\ Monica Fabiana Bento Moreira Thiersch ${ }^{1}$ \\ Argemiro José Moreno Arteaga" $\odot$ \\ Alessandro Araujo Amaral de Almeida"'! \\ Franciane Andrade de Pádual ${ }^{\oplus}$ \\ Cláudio Roberto Thiersch ${ }^{\oplus}$ \\ 'Universidade Federal de São Carlos, Sorocaba, SP, Brasil \\ "Universidade Federal de Campina Grande, Campina Grande, PB, Brasil \\ "'Eucatex, Sorocaba, SP, Brasil
}

\section{RESUMO}

As funções de afilamento são ferramentas úteis para estimar diâmetros de toras e volumes comerciais de madeira a quaisquer alturas, sendo de grande importância para os empreendimentos florestais que buscam a otimização do uso de suas florestas. Este trabalho teve como objetivo avaliar modelos de afilamento segmentados, não segmentados e modelos de forma variável para estudo do afilamento do tronco de árvores de Eucalyptus sp. Com uma base de dados proveniente de um plantio de Eucalyptus sp. localizado no sul do estado de São Paulo aos 6,3 anos de idade, os modelos de afilamento testados foram: os modelos não segmentados de Ormerod (1973) e de Schöepfer (1966), o modelo segmentado de Max e Burkhart (1976), e os modelos de forma variável de Muhairwe (1999), Methol (2001) apud Rachid et al. (2014) e de Kozak (2004). Os volumes reais foram calculados utilizando-se o método de Smalian. O modelo de Kozak (2004) obteve as melhores medidas de precisão para estimativa dos diâmetros, já a equação de Muhairwe (1999) demonstrou os melhores resultados gráficos de dispersão de resíduos para a mesma estimativa. Para a estimativa volumétrica, a equação que obteve os melhores resultados foi a proposta por Methol (2001) apud Rachid et al. (2014), com as melhores medidas de precisão e melhor comportamento gráfico, com menores tendências e dispersão mais concentrada dos resíduos.

Palavras-chave: Multiprodutos; Técnicas de modelagem; Sortimento; Forma do tronco 


\section{ABSTRACT}

Taper functions are useful tools for estimating log diameters and commercial wood volumes at any height, being of great importance to forestry enterprises that aim to optimize the use of their forests. Thus, the aim of this work was to evaluate segmented, non-segmented and variable-exponent taper models for studying the trunk taper of Eucalyptus sp. With a database from a 6.3-year-old Eucalyptus sp. planted forest, in the south of the state of São Paulo, the taper models tested were: the non-segmented models of Schöepfer (1966) and Ormerod (1973) the segmented model of Max and Burkhart (1976), and the variable-exponent models of Muhairwe (1999), Methol (2001) apud Rachid et al. (2014) and Kozak (2004). Real volumes were calculated using the Smalian formula. The equation proposed by Kozak (2004) obtained the best accuracy to estimate the diameters, whereas the Muhairwe (1999) equation showed the best graphic dispersion results for the same estimate. For volume estimation, the equation that obtained the best results was that proposed by Methol (2001) apudRachid et al. (2014), with the best measures of precision and better graphical behavior, with smaller trends and more concentrated dispersion of the residues.

Keywords: Multiproducts; Modeling techniques; Assortment; Stem shape

\section{INTRODUÇÃO}

A demanda mundial crescente por produtos de origem florestal estimula cada vez mais a implantação de novos plantios florestais, de forma a proporcionar a abertura de novos mercados e ampliar ainda mais os horizontes para o setor florestal. As empresas do ramo florestal representam 1,2\% do PIB brasileiro, sendo detentoras dos maiores plantios renováveis do país nos quais a agregação de renda tem cada vez mais importância (INDÚSTRIA BRASILEIRA DE ÁRVORES, 2020). De maneira que um empreendimento, ao se basear na produção de apenas um tipo de produto, está suscetível às oscilações de preço de mercado do mesmo. Sendo assim, uma alternativa seria atender às demandas de múltiplos produtos do mercado da madeira, como serraria, painéis, movelaria, além da celulose, o que garante a rentabilidade da atividade florestal, reduzindo o impacto de grandes oscilações. Nesse contexto, para o planejamento da produção florestal é crucial que esses múltiplos produtos sejam quantificados, possibilitando prever a continuidade e escala no fornecimento de matéria-prima (ASSIS et al., 2002; SOUZA et al., 2008). Para que a quantificação do estoque seja realizada de maneira acurada, são necessários frequentes estudos 
ligados ao volume da árvore, de forma individual ou do povoamento, tornando o uso de modelos matemáticos capazes de representar com acuracidade os perfis do fuste, uma ferramenta de grande importância aos empreendimentos florestais (SOUZA et al., 2008).

O afilamento do fuste (taper) é definido pela taxa de decréscimo do diâmetro (d) ao longo do tronco (HUSCH; BEERS; KERSHAW JUNIOR, 2003). Ao utilizar o diâmetro à altura do peito (DAP, a 1,30 m), a altura total e diferentes alturas ao longo do tronco da árvore, estas equações estimam o diâmetro do tronco a qualquer altura, o volume total do tronco, o volume comercial e altura comercial para qualquer diâmetro a qualquer altura acima do toco e os volumes individuais em qualquer parte, em qualquer altura a partir do solo (KOZAK, 2004).

Conforme indicado por Heidarsson e Pukkala (2011), o afilamento do tronco é um dos vários componentes necessários em um sistema de planejamento de manejo florestal moderno.

Ao integrar uma equação de afilamento obtém-se o volume de madeira existente entre os limites de integração, desse modo, é possível estimar o volume a qualquer altura ao longo do fuste. Esse procedimento é denominado Sortimento e consiste em uma das mais importantes ferramentas do planejamento das florestas de produção, pois permite a maximização do aproveitamento de uma árvore, permitindo a quantificação da matéria-prima de acordo com suas dimensões e qualidade de acordo com os múltiplos usos da madeira (MÜLLER; FINGER; SCHNEIDER, 2005).

Os primeiros modelos de afilamento eram os modelos não segmentados, que se utilizam uma única equação que explica o perfil do fuste, descrevendo-o como um único sólido ou tronco de sólido geométrico, o que pode causar problemas em estimativas próximas à base e no ápice do fuste. Já os segmentados corrigem de certa forma o efeito na base e no ápice, mas apresentam o inconveniente de terem auxílio de restrições (pontos de inflexão) no ajuste dos modelos (MÔRA, 2015).

Para melhorar o desempenho das funções de afilamento quanto ao ajuste e à 
utilização de pontos de inflexão, Kozak (1988) introduziu os modelos de afilamento de forma variável, que empregam modelos de regressão não segmentados que, de forma implícita, dividem a árvore em três segmentos, sem necessidade do auxílio de pontos de inflexão, por possuírem variáveis ou expoentes que variam ao longo do tronco, representando o neiloide, paraboloide, tronco de cone e várias outras formas intermediárias.

Segundo Li e Weiskittel (2010), a maioria das equações de afilamento do tronco publicadas foram desenvolvidas para certas espécies e, frequentemente, seu desempenho é comparado com o de um número limitado de modelos de formatos alternativos. Portanto, é necessário e benéfico estudar mais aprofundadamente as características das equações de afilamento e estender seu uso a outras espécies além daquelas para as quais elas foram originalmente desenvolvidas.

A necessidade da otimização do uso dos recursos florestais, dentro do conceito de múltiplos produtos da madeira, tem justificado e estimulado a realização de estudos sobre o assunto, sendo o uso das funções de afilamento a melhor forma de se determinar o volume entre quaisquer pontos do fuste. Sendo assim, este trabalho tem como objetivo avaliar modelos de afilamento segmentados, não segmentados e de forma variável para estudo do afilamento do tronco de árvores de Eucalyptus sp.

\section{MATERIAL E MÉTODOS}

\subsection{Base de dados}

Os dados deste estudo foram coletados em um plantio de Eucalyptus sp. localizado no sul do Estado de São Paulo, aos 6,3 anos de idade. Para o ajuste das equações propostas foi efetuada a cubagem rigorosa de 163 árvores nas quais foram mensurados os diâmetros com e sem casca nas alturas (hi) absolutas 0,$1 ; 0,7 ; 1,3 ; 2 ; 4$; $6 \mathrm{~m}$ e assim sucessivamente até a altura total $(h)$ de cada árvore. O volume utilizado como volume real foi obtido somando-se os volumes parciais de todas as seções, utilizando-se o método de Smalian. 


\subsection{Modelos matemáticos empregados}

Foram empregadas funções de afilamento segmentadas, não segmentadas e de forma variável para descrever os diâmetros ao longo do fuste (Tabela 1).

Tabela 1 - Equações de afilamento testadas

\begin{tabular}{lcc}
\hline \multicolumn{1}{c}{ Equação } & Referência & $\mathbf{N}^{\circ}$ \\
\hline$d_{i}=\beta_{1} d\left(\frac{h-h i}{h-1,3}\right)^{\beta_{2}}$ & $\begin{array}{c}\text { Ormerod } \\
\text { (1973) }\end{array}$ \\
\hline$d_{i}=d\left[\beta_{1}+\left(\frac{h i}{h}-1\right)+\beta_{2}\left(\left(\frac{h i}{h}\right)^{2}-1\right)+\beta_{3}\left(\alpha_{1}-\frac{h i}{h}\right)^{2} I_{1}+\beta_{4}\left(\alpha_{2}-\frac{h i}{h}\right)^{2} I_{2}\right]^{0,5}$ & $\begin{array}{c}\text { Maxe } \\
\text { Burkhart (1976) }\end{array}$ \\
Sendo: $I_{\mathrm{i}}=1$ se $\alpha_{i} \geq X$, caso contrário: $I_{\mathrm{i}}=0$ &
\end{tabular}

$d_{i}=\alpha_{0} d^{\alpha_{1}} \alpha_{2}{ }^{d}\left(1-\sqrt{\frac{h i}{h}}\right)^{C}$

Sendo: $C=\beta_{1}\left(\frac{h i}{h}\right)+\beta_{2}\left(\frac{h i}{h}\right)^{2}+\beta_{3} /(h i / h)+\beta_{4}\left(\frac{h i}{h}\right)^{3}+\beta_{5} d+\beta_{6}(d / h)$

$d_{i}=\alpha_{0} d^{\alpha_{1}} \alpha_{2}{ }^{d}\left(1-\sqrt{\frac{h i}{h}}\right)^{C}$

Muhairwe

(1999)

Modificada

por

Methol (2001)

apud

Sendo: $C=\beta_{1} \log \left(\frac{h i}{h}+0,001\right)+\beta_{2} e^{\frac{h i}{h}}+\beta_{3} \frac{d}{h}+\beta_{4} \log (d)+\beta_{5} \frac{h}{\sqrt{h i}}+\beta_{6} \frac{d / h}{h i}$

Rachid et al.

(2014)

$d_{i}=\left(\alpha_{0} d^{\alpha_{1}} h^{\alpha 2}\right) m^{c}$

Sendo: $C=\beta_{1}\left(\frac{h i}{h}\right)^{4}+\beta_{2}\left(\frac{1}{e^{d / h}}\right)+\beta_{3} m^{0,1}+\beta_{4} \frac{1}{d}+\beta_{5} h^{\left(1-(h i / h)^{1 / 3}\right)}+\beta_{6} m$

Kozak

$m=\frac{1-\left(\frac{h i}{h}\right)^{1 / 3}}{1-\left(\frac{1,3}{h}\right)^{1 / 3}}$

(2004)

$d_{i}=d\left[\beta_{0}+\beta_{1}\left(\frac{h i}{h}\right)+\beta_{2}\left(\frac{h i}{h}\right)^{2}+\beta_{3}\left(\frac{h i}{h}\right)^{3}+\beta_{4}\left(\frac{h i}{h}\right)^{4}+\beta_{5}\left(\frac{h i}{h}\right)^{5}\right]$

Schöepfer

(1966)

Fonte: Autores (2019)

Em que: $h i=$ altura relativa à secção; $h=$ altura total da árvore; $d_{i}=$ diâmetro nas alturas $h i=(\mathrm{m})$, sendo $i=0,1 ; 0,7 ; 1,3 ; 2 ; 4 ; 6$; assim por diante até a altura total da árvore; $d=\mathrm{DAP} ; \beta_{i}$ e $a_{i}$ parâmetros da regressão. 
As equações foram selecionadas de forma a realizarem-se diversas abordagens, incluindo modelos bem simples como, por exemplo, o modelo proposto por Ormerod (1973), a equação polinomial segmentada desenvolvida por Max e Burkhart (1976), a equação de expoente variável de Muhairwe (1999), uma alteração do expoente desta equação proposta por Methol (2001) apud Rachid et al. (2014), a equação de afilamento de forma variável proposta por Kozak (2004) e o modelo de Schöepfer (1966), que aparece como o modelo de maior uso no Brasil, visto que vem sendo recorrentemente citado por diversos autores (SCOLFORO et al., 1998; FISCHER et al., 2001; ASSIS et al., 2002; MENDONÇA et al., 2007; SOUZA et al., 2008; YOSHITANI JUNIOR et al., 2012; TÉO et al., 2013; MÔRA, 2015).

O processamento dos dados foi realizado com o auxílio do software R (versão 3.2.1) (R CORE TEAM, 2015) e RStudio (versão 0.99.473) (RSTUDIO TEAM, 2015) com o uso da função n/s para o ajuste.

\subsection{Cálculo do volume}

Para o cálculo do volume total e por seção, utilizou-se o procedimento de integração numérica das equações de afilamento. Cada equação foi elevada à potência quadrada e multiplicada pela constante ( $\pi / 40000)$. Essa constante permite a utilização do diâmetro $(d)$ em cm para obtenção do volume $(v)$ em $\mathrm{m}^{3}$. A função é integrada entre dois limites, como na integração definida, adotando 100 subdivisões entre os dois limites usados para integração. A integração foi realizada usando a função integrate associada à função mapply, ambas implementadas no software R.

\subsection{Análise estatística das equações testadas}

Todos os modelos foram ajustados e comparados utilizando: o erro padrão residual em porcentagem (S $\left.S_{y x} \%\right)$, Equação (7), aplicado para ojulgamento da performance de ajuste de equações de regressão por ser a melhor medida para comparar a precisão entre diferentes equações de regressão, em que a melhor equação será a qual se 
observar o menor valor para tal medida de precisão. O "Mean Absolute Bias" (MAB), Equação (8), como uma forma de medir o viés segundo Kozak (2004), calculado como a diferença entre o verdadeiro valor do parâmetro e o valor estimado pela equação, indicando tendenciosidade na equação testada, portanto, quanto menor seu valor, menor a tendência total apresentada pela equação. Além da eficiência do modelo (EF) (PINJUV; MASON; WATT, 2006), Equação (9), que indica a porcentagem da variação da variável dependente explicada pela variável independente, sendo assim, quanto mais próximo for o valor de EF em relação à unidade, melhor terá sido o ajuste.

$$
\begin{aligned}
& S_{y x} \%=\frac{\sqrt{\frac{\sum(Y-\hat{Y})^{2}}{n-p-1}}}{\bar{Y}} \times 100 \\
& M A B=\frac{\sum(|Y-\hat{Y}|)}{n} \\
& E F=1-\frac{\sum(Y-\hat{Y})^{2}}{\sum(Y-\bar{Y})^{2}}
\end{aligned}
$$

Em que: $n=$ número de observações; $Y=$ valor observado; $\hat{Y}=$ valor estimado; $\bar{Y}=$ média geral; $p=$ número de parâmetros do modelo.

Foram realizadas análises gráficas de dispersão dos resíduos e dos gráficos quantil-quantil de normalidade dos resíduos (QQ-plot). A dispersão dos resíduos com a análise de tendência, principalmente indicando em qual região da árvore as tendências são maiores, é de extrema importância para a escolha do melhor modelo, visto que é na parte inferior da árvore que está contida a maior quantidade de madeira, assim sendo, quaisquer tendências nas estimativas resultam em maiores erros. O gráfico quantil-quantil é utilizado para conferir a adequação de distribuição de frequências dos dados a uma distribuição de probabilidades, sendo a normalidade dos resíduos um dos pressupostos da regressão. A análise gráfica fornece mais informações do que simplesmente um teste de normalidade, pois permite explicar a causa da fuga de normalidade sendo: por desvio de assimetria, de curtose, devido à mistura de distribuições, presença de outlier ou aos dados serem discretos. Cada tipo de fuga sugere uma forma de correção como, por exemplo, a deleção de um outlier. 
Os modelos foram ranqueados tanto para estimativa de diâmetro como para estimativa de volume, utilizando as medidas de precisão: $S_{y x} \%$, MAB e EF. O melhor modelo recebeu o ranque 1 e o de pior performance recebeu o ranque 7 , sendo que todas as medidas tiveram o mesmo peso para a definição do ranking geral. Dessa forma, resume-se o comportamento das equações em um único parâmetro, facilitando a visualização dos resultados. Já sob a perspectiva gráfica da dispersão e normalidade dos resíduos, os modelos foram analisados de acordo com seus comportamentos, distribuições, tendências e simetria.

\section{RESULTADOS E DISCUSSÃO}

Na Tabela 2 podem ser observados os parâmetros de regressão estimados para as equações de afilamento em estudo.

Tabela 2 - Parâmetros estimados para as equações de afilamento testadas

\begin{tabular}{lcccccccccc}
\hline Equação & $\boldsymbol{\alpha}_{\mathbf{1}}$ & $\boldsymbol{\alpha}_{\mathbf{2}}$ & $\boldsymbol{\alpha}_{\mathbf{3}}$ & $\boldsymbol{\beta}_{\mathbf{0}}$ & $\boldsymbol{\beta}_{\mathbf{1}}$ & $\boldsymbol{\beta}_{\mathbf{2}}$ & $\boldsymbol{\beta}_{\mathbf{3}}$ & $\boldsymbol{\beta}_{\mathbf{4}}$ & $\boldsymbol{\beta}_{\mathbf{5}}$ & $\boldsymbol{\beta}_{\mathbf{6}}$ \\
\hline$(1)$ & - & - & - & - & 0,71626 & - & - & - & - & - \\
$(2)$ & - & 0,05099 & 0,12487 & - & $-1,53858$ & 0,51364 & 192,8751 & 9,145441 & - & - \\
$(3)$ & 0,84799 & 1,18454 & 0,98240 & - & 0,15506 & $-0,01233$ & $-0,025906$ & 0,015125 & 0,701201 & - \\
$(4)$ & 1,28423 & 1,05171 & 0,99141 & - & $-0,28784$ & 0,22426 & 0,125519 & $-0,00775$ & $-0,006264$ & $-0,15257$ \\
$(5)$ & 1,17996 & 0,93279 & 0,01165 & - & 0,25256 & $-0,62129$ & 0,711660 & 2,075633 & 0,040055 & $-0,31407$ \\
$(6)$ & - & - & - & 1,22949 & $-4,72549$ & 21,4334 & $-48,72881$ & 49,32089 & $-18,52848$ & - \\
\hline
\end{tabular}

Fonte: Autores (2019)

Os pontos de junção $\left(a_{2}\right.$ e $\left.a_{3}\right)$ entre os polinômios obtidos para a equação 3 atende às condições que são citadas em Max e Burkhart (1976), que colocam que a primeira e a segunda derivada dos polinômios devem ser contínuas no ponto de junção entre esses polinômios. Exemplificando, no caso deste trabalho esses pontos encontram-se a aproximadamente 5,1\% e 12,5\% da altura total.

Na Tabela 3, pode-se observar que o modelo que apresentou os melhores resultados para as medidas de precisão nas estimativas dos diâmetros ao longo do fuste foi o modelo proposto por Kozak (2004), com $S_{y x} \%$ de apenas 5,31\% e EF 
de 99,1\%. As equações de Muhairwe (1999) e de Methol (2001) apud Rachid et al. (2014) apresentaram resultados próximos aos da equação de Kozak (2004), porém ligeiramente inferiores, com de aproximadamente 5,37\% e 5,33\%, respectivamente e, além disso, obtiveram valores de eficiência muito próximos a 99,1\%.

Tabela 3 - Análises estatísticas das equações para estimativa dos diâmetros com casca e suas respectivas posições no ranking

\begin{tabular}{lrccc}
\hline \multicolumn{1}{c}{ Equação } & \multicolumn{1}{c}{$\boldsymbol{S}_{\mathbf{y x}}(\%)$} & MAB & EF & Ranking Geral \\
\hline Ormerod & $10,8777(6)$ & $0,618944(6)$ & $0,962011(6)$ & $(6)$ \\
Max \&Burkhart & $5,7459(4)$ & $0,399601(4)$ & $0,989421(4)$ & $(4)$ \\
Muhairwe & $5,3774(3)$ & $0,373009(3)$ & $0,990742(3)$ & $(3)$ \\
Methol & $5,3307(2)$ & $0,366733(1)$ & $0,990906(2)$ & $(2)$ \\
Kozak & $5,3151(1)$ & $0,367225(2)$ & $0,990959(1)$ & $(1)$ \\
Schöepfer & $6,5555(5)$ & $0,473530(5)$ & $0,986230(5)$ & $(5)$ \\
\hline
\end{tabular}

Fonte: Autores (2019)

Entretanto, quando analisados os gráficos de resíduos (Figura 1) e os gráficos de normalidade dos resíduos QQ-plot (Figura 2), o modelo que apresentou os melhores resultados para estimar os diâmetros ao longo do fuste foi o de Muhairwe (1999), já que a distribuição dos resíduos do modelo apenas apresentou uma pequena tendência a superestimar os diâmetros próximos à linha do solo, abaixo de $5 \mathrm{~m}$, permanecendo mais estável, ao contrário dos demais modelos testados que apresentaram algum tipo de tendência ao longo do fuste.

Os modelos que apresentaram os melhores valores das medidas de precisão tiveram um comportamento de resultado inferior na distribuição dos seus resíduos, tendo o modelo de Kozak (2004) apresentado tendência a superestimar os diâmetros nas menores alturas, até $6 \mathrm{~m}$, e subestimar no meio do fuste, entre 6 e 14 m; já o modelo proposto por Methol (2001) apud Rachid et al. (2014), além de ter uma pequena tendência a superestimar os diâmetros nas menores alturas, até $5 \mathrm{~m}$, e também entre 10 e 15 m, ainda subestimou os diâmetros nas maiores alturas, acima de 20 m. Os QQplot para esses modelos afirmam a normalidade dos resíduos e demonstram resultados superiores às demais equações pelo comportamento simétrico, principalmente nas regiões centrais do fuste, acompanhando a reta normal, devido à maior acuracidade nas estimativas dos diâmetros. 
Figura 1 - Distribuição dos resíduos (cm) ao longo das alturas relativas (hi) para estimativa dos diâmetros pelos ajustes das equações de afilamento

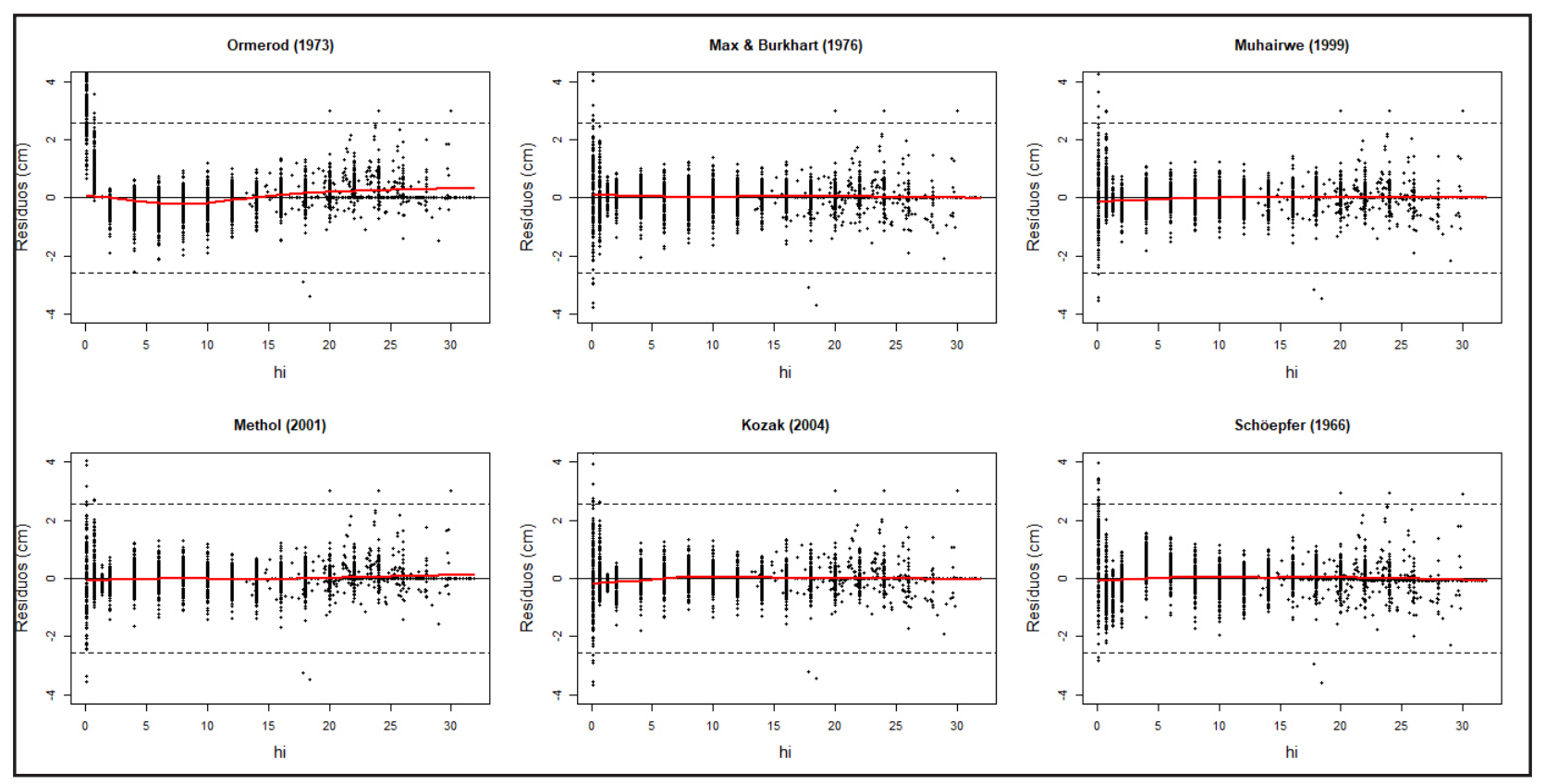

Fonte: Autores (2019)

Em que: * A linha vermelha representa a linha de tendência de cada modelo, já a linha pontilhada é o limite determinado pela distribuição de $t$ para um intervalo de confiança de $1 \%$.

Figura 2 - QQ-plot da normalidade dos resíduos para cada modelo de afilamento ajustado para estimativa dos diâmetros ao longo do fuste

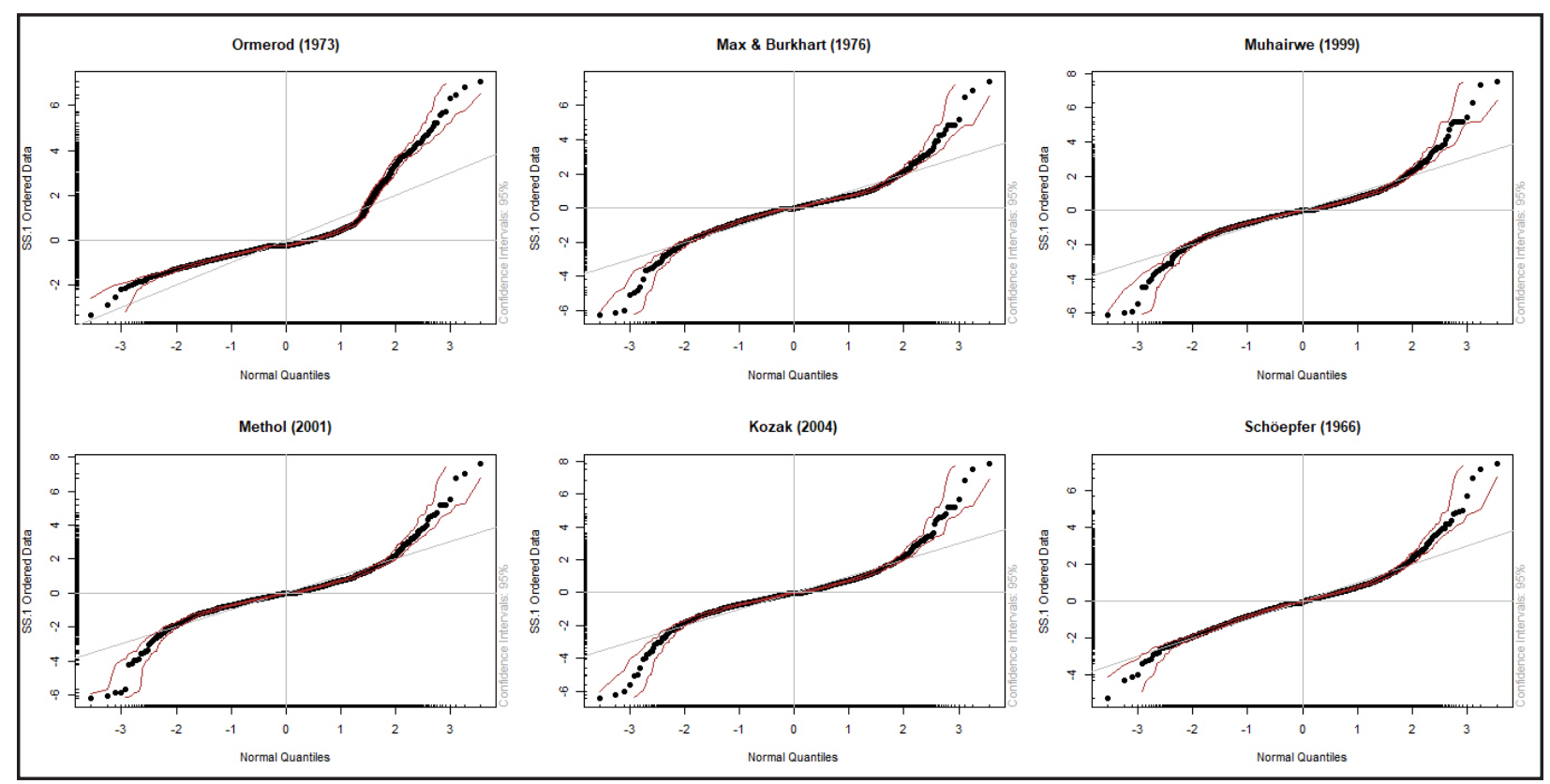

Fonte: Autores (2019) 
O modelo de Max e Burkhart (1976) apresentou a $4^{\mathrm{a}}$ melhor colocação para as medidas de precisão com $S_{y x} \%=5,74$ e EF = 98,94\%, porém seu comportamento gráfico apresenta-se inferior aos melhores modelos devido às tendências em subestimar os diâmetros entre 7 e 16 m de altura e superestimar os acima de 22 m.

Rachid et al. (2014) apontaram que, em geral, em se tratando de equações de afilamento, os modelos mais simples têm performances inferiores aos modelos mais complexos, sendo assim, os resultados observados neste trabalho se apresentaram conforme o esperado, pois o modelo de Ormerod (1973) apresentou o pior resultado para as estimativas dos diâmetros ao longo do fuste, tanto sob a perspectiva das medidas de precisão quanto sob as análises dos gráficos de resíduos e tendências (Figura 1). Esse modelo apresentou comportamento assimétrico para a dispersão de resíduos, superestimando os valores de diâmetros até 1,3 m e subestimandoos nas maiores alturas a assimetria observada no gráfico QQ-plot, que confirma os resultados estatísticos demonstrando grande assimetria pelos pontos dispostos em forma de arco.

Quanto ao modelo de Schöepfer (1966), apesar de ter apresentado resultados considerados aceitáveis, como $S_{y x} \%=6,55 \%$ e $E F=98,62 \%$, ficou apenas com a $5^{a}$ colocação no ranking das equações e apresentou problemas de tendência, subestimando os diâmetros entre 5 e $22 \mathrm{~m}$ e superestimando os diâmetros abaixo de $5 \mathrm{~m}$.

Os volumes estimados das seções ao longo do fuste demonstram que nem sempre o melhor modelo para o ajuste dos diâmetros será o melhor modelo para a estimativa dos volumes, visto que para o cálculo de volume a equação que obteve os melhores resultados foi a proposta por Methol (2001) apud Rachid et al. (2014), como pode ser observado na Tabela 4 e na Figura 3. Isso pode ser explicado pelo fato de alguns modelos apresentarem tendências maiores ao estimar os diâmetros em regiões específicas da árvore que resultam em maiores erros, como por exemplo, nas menores alturas, nas quais o volume de madeira é maior e, por isso, o menor erro no cálculo dos diâmetros já resulta em perda de qualidade ao se estimarem os volumes. 
Tabela 4 - Análises estatísticas das equações para estimativa dos volumes com casca

\begin{tabular}{lcccc}
\hline \multicolumn{1}{c}{ Equação } & $\boldsymbol{S}_{\mathbf{y x}}(\%)$ & MAB & EF & Ranking Geral \\
\hline Ormerod & $6,3455(5)$ & $0,0065181(6)$ & $0,993694(5)$ & $(5)$ \\
Max e Burkhart & $6,3594(6)$ & $0,0062126(5)$ & $0,993667(6)$ & $(6)$ \\
Muhairwe & $4,6683(3)$ & $0,0047588(3)$ & $0,996587(3)$ & $(3)$ \\
Methol & $4,6415(1)$ & $0,0046640(1)$ & $0,996626(1)$ & $(1)$ \\
Kozak & $4,6523(2)$ & $0,0046890(2)$ & $0,996611(2)$ & $(2)$ \\
Schöepfer & $6,2113(4)$ & $0,0062124(4)$ & $0,993958(4)$ & $(4)$ \\
\hline
\end{tabular}

Fonte: Autores (2019)

De maneira geral, as três melhores equações, de Muhairwe (1999), Methol (2001) apud Rachid et al. (2014) e Kozak (2004), são recomendadas pelos erros inferiores a 5\%, pela alta eficiência (aproximadamente 99,7\%), e pelo comportamento gráfico para a dispersão dos resíduos. Os modelos de Muhairwe (1999) e Kozak (2004) apresentaram distribuições dos resíduos mais concentradas (Figura 3) com uma pequena tendência a subestimar os volumes a partir dos 10 e 15 m, respectivamente, até a altura total. Já o modelo proposto por Methol (2001) apud Rachid et al. (2014) apresenta uma distribuição um pouco menos concentrada, porém quase sem tendências, mantendose estável ao longo do fuste.

Os resultados observados para o modelo de Methol (2001) apud Rachid et al. (2014) perante a análise da normalidade dos resíduos pelos gráficos de QQ-plot (Figura 4) apresentam-se mais simétricos e próximos à reta quando comparados aos dos demais modelos, com exceção ao modelo de Kozak (2004) que também apresentou um bom comportamento da normalidade dos resíduos. O modelo de Muhairwe (1999) apresentou forte assimetria por fuga nos extremos do gráfico QQ-plot; os modelos de Schöepfer (1966), Ormerod (1973) e o de Max e Burkhart (1976) apresentaram arqueamento dos resíduos.

O modelo de Maxe Burkhart (1976), que havia apresentado um ótimo ajuste para o cálculo dos diâmetros, obteve resultados muito inferiores para o cálculo de volume, caindo da $4^{\mathrm{a}}$ para a $6^{\mathrm{a}}$ posição no ranking, alcançando valores muito semelhantes aos da equação de Ormerod (1973), de formato muito mais simples. 
O modelo de Schöepfer (1966), apesar de ter se apresentado como o $4^{\circ}$ melhor modelo para estimativa de volume segundo o ranking proposto, encontra-se com valores mais próximos aos piores modelos. Ao se analisar seu comportamento quanto à dispersão dos resíduos este modelo demonstrou grandes problemas de tendência a subestimar os volumes em quase toda a extensão do fuste, o que o torna um modelo inferior quando comparado com o de Methol (2001) apud Rachid et al. (2014), por exemplo.

Figura 3 - Distribuição dos resíduos $\left(\mathrm{m}^{3}\right)$ ao longo das alturas (hi) para as estimativas volumétricas a partir da integração das equações de afilamento

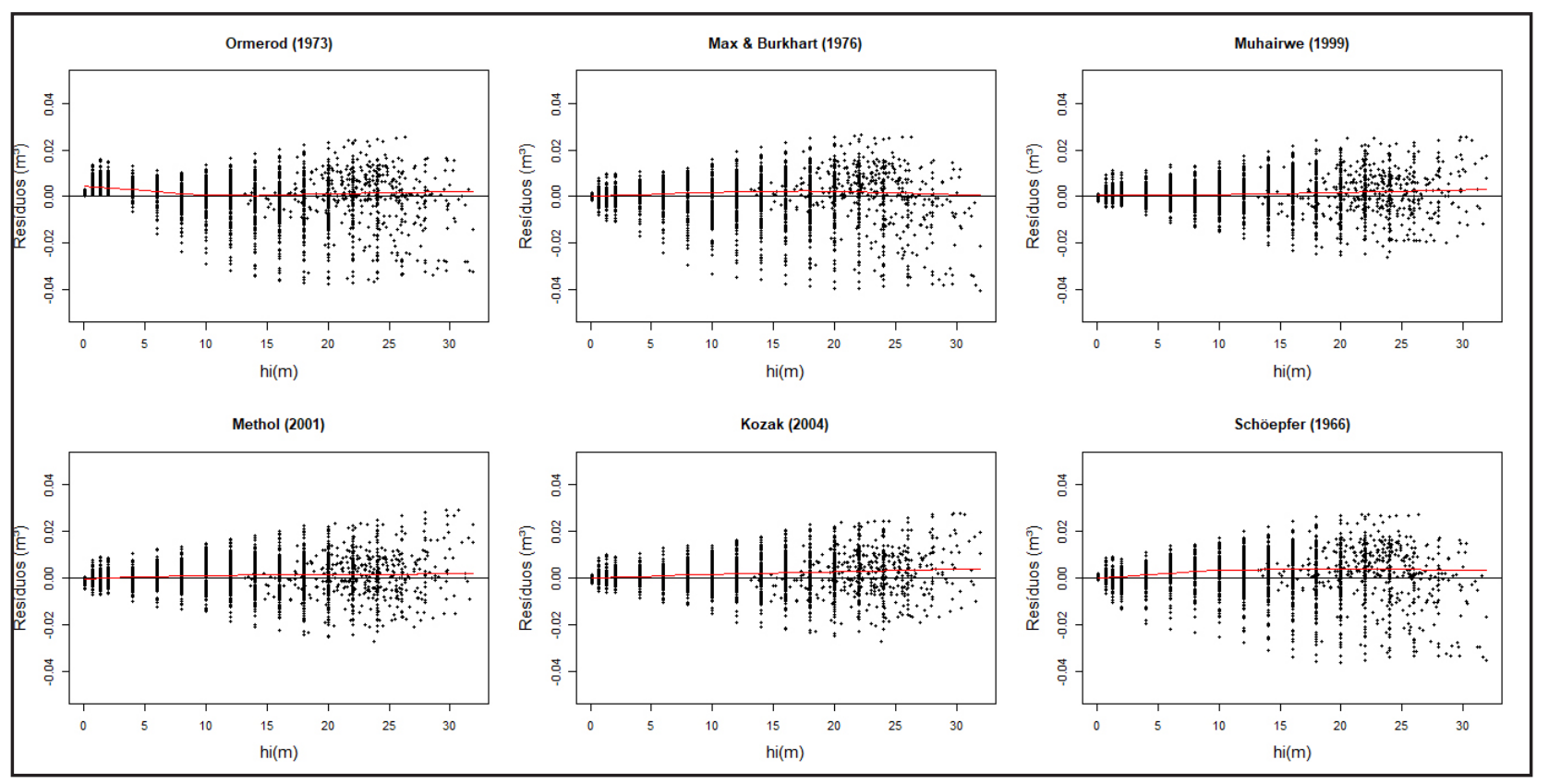

Fonte: Autores (2019)

Em que: * A linha vermelha representa a linha de tendência de cada modelo.

Özçelik e Crecente-Campo (2016), estudando afilamento do tronco de Cedruslibani A. Rich., observaram que, apesar de amplamente utilizada e reconhecida por ser uma das melhores equações de afilamento, e ter se ajustado bem à base de dados em questão, a equação de forma variável de Kozak (2004) não mostrou o melhor ajuste, apresentando-se inferior aos modelos segmentados de Clark, Souter 
e Schlaegel (1991), Bi (2000) e Fang, Borders e Bailey (2000) para as estimativas de diâmetro e altura. Entretanto, para a estimativa de volume, o modelo proposto por Kozak (2004) apresentou resultados melhores, ficando atrás apenas dos modelos de Clark, Souter e Schlaegel (1991) e Fang, Borders e Bailey (2000).

Figura 4 - QQ-plot de normalidade dos resíduos para cada modelo de afilamento testado para estimativa de volume

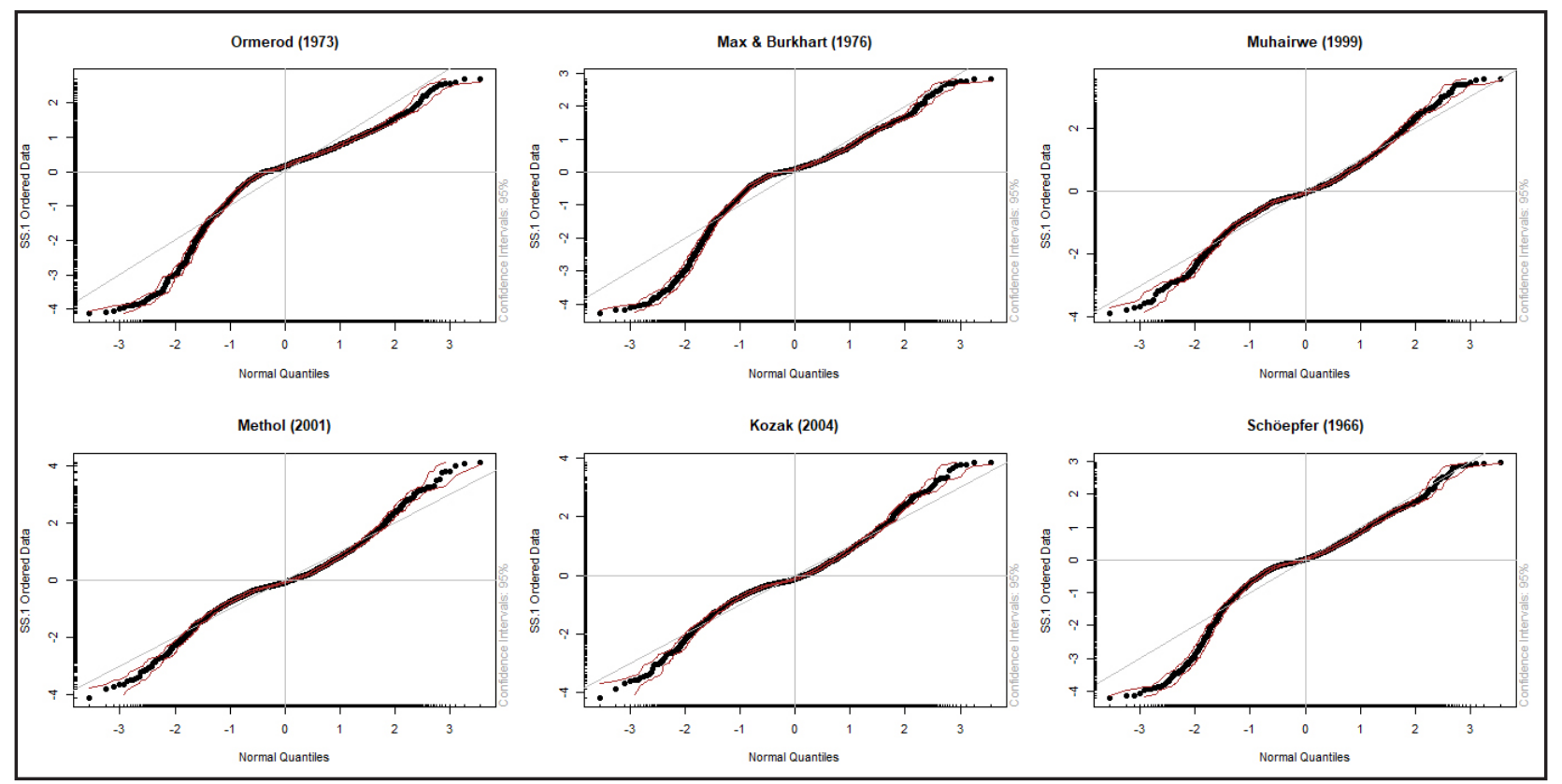

Fonte: Autores (2019)

Como apontado por Li e Weiskittel (2010), quando existe a possibilidade de mensurar os diâmetros a 5,30 m, o modelo de Clark, Souter e Schlaegel (1991) se apresenta como o melhor, contudo, não são em todos os casos que essa medida pode ser obtida, principalmente de forma não destrutiva. Quando essa medida não pode ser aferida, os modelos de forma variável se apresentam como uma alternativa muito eficiente, como pode ser observado nos resultados obtidos pelos mesmos autores, que apontaram a equação de Kozak (2004) como a melhor equação para estimar o afilamento do tronco de duas das três espécies estudadas. 
Sendo assim, os resultados obtidos no presente estudo estão de acordo com os observados na literatura, visto que os melhores modelos para o afilamento do tronco de Eucalyptus sp. foram os de forma variável de Muhairwe (1999), Methol (2001) apud Rachid et al. (2014) e Kozak (2004) devido aos melhores valores para as medidas de precisão testadas e à melhor dispersão de resíduos e pouca tendência.

\section{CONCLUSÕES}

As equações de forma variável apresentaram os melhores resultados para as medidas de precisão e para o comportamento gráfico nas estimativas de diâmetro e volume em comparação às equações segmentadas e não segmentadas.

A equação de Kozak (2004), Equação (5), apresentou as melhores medidas de precisão para a estimativa dos diâmetros, porém a equação que apresentou a melhor dispersão dos resíduos foi a equação de Muhairwe (1999), Equação (3). A dispersão dos resíduos e a linha de tendência são os fatores mais importantes para a escolha da melhor equação devido ao fato de equações menos tendenciosas serem mais confiáveis.

A equação proposta por Methol (2001) apud Rachid et al. (2014), Equação (4), obteve os melhores valores para as medidas de precisão e ainda a melhor distribuição dos resíduos e menor tendência ao estimar os volumes ao longo do fuste. Sendo assim, novos estudos com diferentes bases de dados devem ser realizados para observar o comportamento desta equação, para confirmar a regularidade de sua eficácia. Seja para diferentes gêneros ou mesmo apenas para o caso do gênero Eucalyptus.

Apesar da sua ampla utilização nos inventários florestais brasileiros, o modelo de Schöepfer (1966), Equação (6), apresentou performance inferior quando comparada às equações de forma variável. 


\section{AGRADECIMENTOS}

O presente trabalho foi realizado com apoio da Coordenação de Aperfeiçoamento de Pessoal de Nível Superior - Brasil (CAPES) - Código de Financiamento 001.

\section{REFERÊNCIAS}

ASSIS, A. L. et al. Avaliação de modelos polinomiais segmentados e não segmentados na estimativa de diâmetros e volumes comerciais de Pinus taeda. Ciência Florestal, Santa Maria, v. 12, n. 1, p. 89-107, 2002.

$\mathrm{BI}, \mathrm{H}$. Trigonometric variable-form taper equations for Australian Eucalyptus. Forest Science, Washington, v. 46, n. 3, p. 397-407, 2000.

CLARK, A. III; SOUTER, R. A.; SCHLAEGEL, B. E. Stem profile equations for southern tree species. [S. I.]: USDA Forest Service, 1991. 113 p. (Research Paper SE-282).

FANG, Z.; BORDERS, B. E.; BAILEY, R. L. Compatible volume taper models for loblolly and slash pine based on a system with segmented-stem form factors. Forest Science, Oxford, v. 46, p. $1-12,2000$.

FISCHER, F. et al. Exatidão dos modelos polinomiais não-segmentados e das razões entre volumes para representar o perfil do tronco de Pinus taeda. Ciência Florestal, Santa Maria, v. 11, n. 1, p. 167-188, 2001.

HEIDARSSON, L.; PUKKALA, T. Taper functions for lodgepole pine (Pinus contorta) and Siberian larch (Larix sibirica) in Iceland. Icelandic Agricultural Sciences, Borgarnes, v. 24, n. 1, p. 3-11, 2011.

HUSCH, B.; BEERS, T. W.; KERSHAW JUNIOR, J. A. Forest mensuration. 4th ed. Hoboken: John Wiley \& Sons, 2003. 443 p.

INDÚSTRIA BRASILEIRA DE ÁRVORES. Relatório anual IBÁ 2020. São Paulo, 2020. Disponível em: https://iba.org/datafiles/publicacoes/relatorios/relatorio-iba-2020.pdf. Acesso em: 23 dez. 2020.

KOZAK, A. My last words on taper equations. The Forestry Chronicle, Mattawa, v. 80, n. 4, p. 507-515, 2004.

KOZAK, A. A variable-exponent taper equation. Canadian Journal of Forest Research, Ottawa, v. 18, n. 11, p. 1363-1368, 1988.

LI, R.; WEISKITTEL, A. R. Comparison of model forma for estimating stem taper and volume in the primary conifer species of the North American Acadian Region. Annals of Forest Science, Les Ulis, v. 67, p. 302-317, 2010.

MAX, T. A.; BURKHART, H. E. Segmented polynomial regression applied to taper equations. Forest Science, Washington, v. 22, n. 3, p. 283-289, 1976. 
MENDONÇA, A. R. et al. Avaliação de funções de afilamento visando a otimização de fustes de Eucalyptus sp. para multiprodutos. Cerne, Lavras, v. 13, n. 1, p. 71-82, 2007.

MÔRA, R. Funções de afilamento de forma variável e modelagem de efeitos mistos em fustes de Pinus taeda e Eucalyptus saligna. 2015. Tese (Doutorado em Engenharia Florestal) - Universidade Federal do Paraná, Curitiba, 2015.

MUHAIRWE, C. K. Taper equations for Eucalyptus pilularis and Eucalyptus grandis for the north coast in New South Wales, Australia. Forest Ecology and Management, Amsterdam, v. 113, n. 2/3, p. 251-269, 1999.

MÜLLER, I.; FINGER, C. A. G.; SCHNEIDER, P. R. Forma de tronco e sortimentos de madeira de Eucalyptus grandis (Hill ex. Maiden), na Região Sudeste do Estado do Rio Grande do Sul. Ciência Florestal, Santa Maria, v. 15, n. 3, p. 293-305, 2005.

ORMEROD, D. W. A simple bole model. The Forestry Chronicle, Mattawa, v. 49, n. 3, p. 136$138,1973$.

ÖZÇELIK, R.; CRECENTE-CAMPO, F. Stem taper equations for estimating merchantable volume of Lebanon Cedar Trees in the Taurus Mountains, Southern Turkey. Forest Science, Oxford, v. 62, n. 1, p. 78-91, 2015.

PINJUV, G.; MASON, E. G.; WATT, M. Quantitative validation and comparison of a range of forest growth model types. Forest Ecology and Management, Amsterdam, v. 236, n. 1, p. 37-46, 2006.

RACHID, C. C. et al. Volume and Taper Equations for P. taeda (L.) and E. grandis (Hill ex. Maiden). Agrociencia Uruguay, Montevideo, v. 18, n. 2, p. 47-60, 2014.

R CORE TEAM. R: a language and environment for statistical computing. Vienna: R Foundation for Statistical Computing, 2015. Disponível em: http://www.R-project.org/. Acesso em: 20 jan. 2021.

RSTUDIO TEAM. RStudio: integrated development for R. Boston, 2015. Disponívelem: http:// www.rstudio.com/. Acesso em: 20 jan. 2021.

SCHÖEPFER, W. Automatisierung des Massen, Sorten und Wertberechnungstenender Waldbestande Schriftenreihe Bad.[S. I.]: Wurtt-Forstl, 1966.

SCOLFORO, J. R. S. et al. Acuracidade de equações de afilamento para representar o perfil do fuste de Pinus elliottii. Cerne, Lavras, v. 4, n. 1, p. 100-122, 1998.

SOUZA, C. A. M. et al. Avaliação de modelos de afilamento não-segmentados na estimação da altura e volume comercial de Eucalyptus sp. Ciência Florestal, Santa Maria, v. 18, n. 3, p. 387399, 2008.

TÉO, S. J. et al. Modelos de Afilamento para Pinus elliottii em diferentes idades, na região de caçador, SC. Floresta, Curitiba, v. 43, n. 3, p. 439-452, 2013.

YOSHITANI JUNIOR, M. et al. Funções de afilamento para plantios desbastados de Pinus taeda. Floresta, Curitiba, v. 42, n. 1, p. 169-176, 2012. 


\section{Contribuição de Autoria}

\section{1 - Lucas Kröhling Bernardi}

Engenheiro Florestal, Me., Doutorando

https://orcid.org/0000-0002-7199-989X•bernardilucas@estudante.ufscar.br

Contribuição: Conceituação, Análise Formal, Curadoria de dados, Metodologia, Investigação, Visualização de dados, Software, Escrita - primeira redação

\section{2 - Monica Fabiana Bento Moreira Thiersch}

Engenheira Agrícola, Dra., Professora

https://orcid.org/0000-0001-8039-0253•monicathiersch@ufscar.br

Contribuição: Administração do projeto, Supervisão, Escrita - revisão e edição, Análise Formal

\section{3 - Argemiro José Moreno Arteaga}

Engenheiro Ambiental, Me., Doutorando

https://orcid.org/0000-0002-4700-3263•argemor29@hotmail.com

Contribuição: Validação, Escrita - revisão e edição

\section{4 - Alessandro Araujo Amaral de Almeida}

Engenheiro Florestal, Me., Analista de Planejamento e Inventário Florestal https://orcid.org/0000-0002-0764-7558• aalmeidaalessandro@gmail.com Contribuição: Investigação, Recursos, Validação

\section{5 - Franciane Andrade de Pádua}

Engenheira Florestal, Dra. Professora

https://orcid.org/0000-0002-3349-8648•franciane@ufscar.br

Contribuição: Obtenção de financiamento, Supervisão, Escrita - revisão e edição

\section{6 - Cláudio Roberto Thiersch}

Engenheiro Florestal, Dr. Professor

https://orcid.org/0000-0003-1558-3168•crthiersch@ufscar.br

Contribuição: Supervisão, Investigação, Análise Formal, Curadoria de dados, Validação, Software, Conceitualização, Escrita - revisão e edição 


\section{Como citar este artigo}

Bernardi, L. K.; Thiersch, M. F. B. M.; Arteaga, A. J. M.; Almeida, A. A. A.; Pádua, F. A.; Thiersch, C. R. Diferentes modelos para o afilamento do tronco de Eucalyptus sp. para o cenário florestal brasileiro. Ciência Florestal, Santa Maria, v. 31, n. 3, p. 1364-1382, 2021. DOI 10.5902/1980509840376. Disponível em: https://doi.org/10.5902/1980509840376. Acesso em: xx mês-abreviado. 2021. 